\title{
La guérison en cancérologie : une improbable ambition
}

\section{Cure in cancer: an improbable ambition}

\section{Marie Ménoret}

Université Paris 8. Centre de Recherches Sociologiques et Politiques de Paris. Paris, France.

E-mail : marie.menoretळcnrs.fr

\section{Courrier}

Centre de Recherches Sociologiques et Politiques de Paris. UMR 7217. Rue Pouchet, 59. Paris, France. 75017.

\section{Résumé}

Cet article propose une réflexion sur la notion de guérison et sur ses usages en cancérologie. Un premier point ausculte sa mise en œuvre en tant qu'ambition à la fois médicale et politique suprême de la mission fondatrice de la cancérologie française. Un deuxième axe pointe ses divers accommodements sociaux, en observant les différentes figures et mésaventures du concept confronté à l'épreuve de l'incurabilité d'un certain nombre de cancers. Enfin, une troisième partie de l'article s'intéresse aux effets de l'incertitude chronique inhérente à l'oncologie. Fondée sur des recherches empiriques inspirées par la grounded theory, par l'interactionnisme et sous l'influence revendiquée d'Anselm Strauss, cette réflexion peut être vue comme un abrégé des travaux de l'auteure, réalisés de la fin des années 1990 à nos jours, sur la cancérologie française. L'incertitude médicale et sa gestion, individuelle et collective, sont au cœur de ce travail.

Mots-clés: Sociologie; Cancer; Guérison; Rémission; Incertitude Médicale. 


\section{Abstract}

This article proposes a reflection about the notion of cure and its uses in oncology. A first point examines its implementation as the utmost medical and political ambition of the founding mission of French oncology. A second axis points to its various social accommodations, observing the different figures and misadventures of the concept confronted with the test of the incurability of numerous cancers. Finally, a third part of the article focuses on the effects of the chronic uncertainty inherent in oncology. Based on empirical research made from the late 1900 s to our days, inspired by grounded theory, interactionism and under the influence of Anselm Strauss, this reflection can be seen as an abstract of the author's work on French oncology. Medical uncertainty and its individual and collective management are at the core of this work.

Keywords: Sociology; Cancer; Curability; Remission; Medical Uncertainty.

\section{La guérison en cancérologie : une improbable ambition'}

Figure I - L'affaire Tournesol
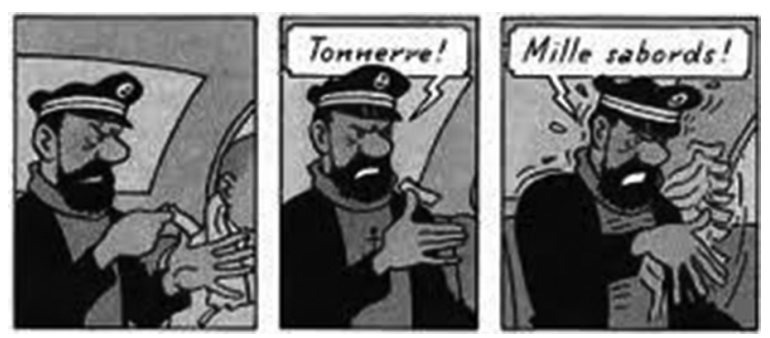

Source : Hergé, 1956, p. 45

Imaginons un instant - exercice de médecinesociologie-science-fictions - que le cancer soit une affection bénigne : l'épreuve de cette maladie serait sans aucun doute autrement vécue par les uns et les autres et autrement approchée par les disciplines qui s'y consacrent. Rarement traité en tant que tel, le sujet de la guérison se révèle pourtant un filtre fécond pour contraster à la fois l'évolution de la médecine moderne et celle des relations entre soignants et soignés. C'est à ce truisme que s'intéresse cet article en proposant d'éclairer la toile de fond de la sociologie du cancer et des sujets qui y sont liés : l'incertitude. Elle enrobe - au moins depuis l'avènement de la médecine clinique - tout diagnostic de cancer et toute tentative de l'éradiquer. Elle est inscrite dans de multiples expectatives au cœur des mondes du cancer : intime et privé, collectif et social, professionnel et politique. Le sens d'expectative retenu ici est son sens premier : une expectative désigne littéralement une attente fondée sur des promesses ou sur des probabilités. C'est sur ce dispositif de promesses et de probabilités que fonctionne la cancérologie. La promesse, c'est celle que tout va être fait pour sauver le/la malade. La probabilité, c'est l'argument qui outille la promesse. Incertitude et expectative imprègnent toute expérience du cancer : de la plus personnelle à la plus publique.

Je l'ai montré depuis le début de mes travaux empiriques sur la cancérologie en m'inspirant des modèles de trajectoires de maladie d'Anselm Strauss :

\footnotetext{
1 Cet article est issu d'une recherche financée par l'Institut National du Cancer (2013-2016) intitulée « Survivre à un cancer : Sociologie d'une condition chronique ».
} 
s'engager dans une prise en charge thérapeutique difficile, mettant en œuvre des traitements lourds, plus ou moins mutilants ou iatrogènes, tout cela pour un objectif dont l'issue est incertaine, ne relève pas d'une simple sinécure (Ménoret, 1999). La cancérologie n'ayant encore atteint nulle part les objectifs d'éradication totale qu'ambitionnait le National Cancer Act de 1971 (Rettig, 1977), la doxa médicale a modifié progressivement son discours sur la guérison. Mais les métamorphoses de ses différentes figures de guérison, telles qu'on les a vues se décliner depuis la fin du $19^{\text {ème }}$ siècle avec le développement de la cancérologie moderne illustrent une constante : on ne peut se débarrasser de l'incertitude qui entoure la notion de guérison dans le monde - dans les mondes - du cancer. Cette incertitude qui, comme le sparadrap du Capitaine Haddock, colle à toutes les images du cancer même les plus encourageantes et, justement, prometteuses, est au cœur de cet article.

Je vais en étayer un développement problématique en développant successivement trois thèmes qui se croisent autour du sujet. Le premier suit la mise en œuvre de la guérison comme ambition suprême avec la mission fondatrice de la cancérologie française. Le second pointe ses divers accommodements avec le réel en observant les figures et mésaventures du concept. Le troisième s'intéresse aux effets de l'incertitude chronique sur la guérison des cancers.

\section{La guérison comme ambition}

La notion de guérison est différemment mobilisée, voire revendiquée, selon les catégories d'acteurs qui la sollicitent. Entre une guérison basée sur la preuve médicale et une autre basée sur l'expérience des personnes, on embrasse un certain nombre de débats ou polémiques qui agitent les controverses autour des médecines alternatives et complémentaires depuis des décennies : preuve médicale contre preuve anthropologique, la guérison des épidémiologistes et celle des patient.e.s ou ex-patient.e.s. Ce rappel nous signifie concrètement - si besoin était - qu'on repère autour de la guérison tout un système de valeurs, de croyances, de normes. Qu'on y distingue également un vaste registre d'enjeux - professionnels ou autres. Qu'on y observe enfin un monde d'interactions complexes dans le travail que constitue l'activité du guérir. Normes, enjeux, interactions : ces trois axes majeurs qui organisent une grande partie de la recherche SHS en santé (Carricaburu; Ménoret, 2004) sont exploitables autour du sujet de la guérison.

Canguilhem (2002) dans un texte intitulé «Une pédagogie de la guérison est-elle possible? » publié au printemps de 1978 - dans une revue de psychanalyse - comparait deux figures du soin : celle du médecin et celle du guérisseur. Deux figures publiques qui non seulement n'ont pas la même légitimité mais l'établissent fort différemment sur le terrain, précisément, de la guérison. Selon Canguilhem, le médecin reste médecin même lorsqu'il ne guérit pas - car son statut est établi sur ses titres, euxmêmes distingués sur des connaissances, pas sur ses résultats - alors que le guérisseur doit en permanence faire la preuve de son statut parce que ce statut, à la différence du médecin, ne repose pas sur ses connaissances mais sur ses réussites. Pour le médecin et pour le guérisseur, le rapport à la guérison est inverse. Le médecin est habilité à prétendre guérir, alors que c'est la guérison telle qu'elle est vécue par le patient/client/usager qui atteste de la solvabilité du travail du guérisseur. C'est dans cette configuration spéciale que la guérison peut se contenter d'être une ambition inachevée. Sans que cet état de fait nuise à l'élaboration de sa revendication en termes d'excellence ou d'évaluation. Remarquablement, sa définition reste une gageure depuis plus d'un siècle.

\section{Une définition impossible d'usage}

La guérison en cancérologie, c'est une vieille question, un vieux problème qui est abordé dès les premières thèses soutenues par des apprentis cancérologues, comme en atteste celle présentée pour le doctorat en médecine en 1929 à la Faculté de médecine de Paris (Lainé, 1929). Lorsque son auteur tente d'évaluer les premiers résultats obtenus par radiothérapie dans un centre régional à partir d'un certain nombre de cas observés dans le cadre de son activité, il écrit :

Nous aurions désiré présenter dans nos statistiques les proportions des malades actuellement guéris. Mais les derniers examens de beaucoup d'entre 
eux n'étant pas suffisamment récents, et nous trouvant dans l'impossibilité d'obtenir de tous par correspondances des précisions sur leur état actuel, nous avons dû nous borner à indiquer, avec le chiffre des malades traités au cours de chaque année, le nombre des survies actuelles comprenant outre les guérisons, les malades prolongés par le traitement dans une mesure que les dates permettront d'apprécier.

Aucune précision n'est mentionnée dans sa thèse - ni méthodologique ni épistémologique qui permettrait de saisir les critères de définition médicale de la guérison. Les expressions retenues pour apprécier les résultats des thérapeutiques oscillent entre : "reste intégralement guérie à cette date", "malade toujours en excellent état", "persistance de la guérison", "une parente apporte d'elle les meilleures nouvelles", "la guérison se maintient actuellement", ou bien encore : "l'état de guérison apparent est de nouveau vérifié et se maintient trois ans et demi après le début du traitement radioactif".

Cette thèse permet de situer le problème de la guérison tel qu'il se pose à cette époque : si la statistique est déjà depuis quelque temps en train de faire ses preuves sur le terrain de la santé, plus précisément de la santé publique, en revanche l'évaluation des thérapies en est encore à ses balbutiements.

\section{Un outil d'évaluation}

Or nous sommes, dans ces années 1920, au cœur d'un conflit professionnel majeur qui est celui qui oppose les chirurgiens aux radiothérapeutes (Pinell, 1992). Jusque-là, les chirurgiens étaient les seuls spécialistes du cancer et ils n'ont pas l'intention de perdre cette hégémonie en faveur des radiothérapeutes qui ont commencé à utiliser la découverte de Röntgen ${ }^{2}$ dès la fin du $19{ }^{\text {ème }}$. Qui guérit? Qui guérit le mieux ? La réponse à cette question est susceptible de déterminer laquelle de ces spécialités peut être déclarée la plus légitime sur le terrain de l'activité médicale. Si l'on voit à la lecture de la thèse citée plus haut que sa définition et son calcul sont encore assez incertains, on devine aussi les enjeux qui se tiennent derrière les chiffres. La guérison devient dès le début du $2 \mathrm{O}^{\text {ème }}$ siècle un argument majeur pour justifier telle ou telle pratique en cancérologie même s'il s'agit d'un calcul difficile à établir. Pour l'expert censé la définir et la préciser, pour le patient qui ne sait pas toujours à quels chiffres se vouer et, enfin, pour le cancérologue lui-même qui n'est pas toujours à l'aise dans la présentation de ces taux à un patient en consultation. Parce que la définition de la guérison en cancérologie fait appel à la moyenne et à la probabilité. Et que passer de la moyenne au cas individuel constitue une tâche délicate. Ce premier usage de la notion de guérison pour justifier une pratique, pour évaluer une méthode : en l'occurrence la chirurgie par rapport à la radiothérapie et réciproquement, affine le propos de Canguilhem selon lequel les connaissances priment sur les résultats quand on est médecin. Il confirme aussi celui de Porter (1996) selon lesquels « les chiffres, les courbes et les formules » sont à considérer « avant tout comme la base de stratégie de communication ».

Un deuxième thème entre en jeu dans la problématique de la guérison du cancer, à cette époque, à côté de celui de la justification de telle ou telle option thérapeutique. Celui-ci se situe cette fois au niveau de l'organisation sociale et politique de la santé publique (Berlivet, 2013) : la guérison se trouve en effet au cœur de l'argument concernant l'institutionnalisation des soins anticancéreux dans les années 1920.

La Première guerre mondiale avait donné l'occasion aux pouvoirs publics d'établir un état des lieux de la morbidité en France et de constater que le cancer n'avait rien à envier à la tuberculose en termes d'impact sur les populations. En 1922, une commission du cancer est donc mise en place auprès de Paul Strauss qui est alors ministre de l'hygiène, de l'assistance et de la prévoyance sociale et qui élève la lutte contre le cancer au rang des causes nationales. Cette commission fait appel à Jean-Alban Bergonié afin de construire une politique de santé publique anticancéreuse. 
L’argument économique prend une importance capitale dans la justification des efforts publics nécessaires à la mise en place du dispositif spécialisé. Pour résumer, le traitement du cancer est en train de devenir une médecine de haute technologie et le futur centre anticancéreux s'annonce comme la structure hospitalière la plus coûteuse que l'on ait jusqu'alors connue. Il importe donc d'argumenter efficacement la rentabilité de l'entreprise. Compte tenu de la répartition à cette époque de la clientèle entre secteur privé et secteur public, cette rentabilité ne peut être envisagée en termes purement lucratifs pour les institutions puisque les centres anticancéreux, selon les conventions initiales proposées, ne recevront que des malades non-payants. Les investissements nécessaires pour soigner ces malades vont donc être justifiés sur des arguments de «valeur sociale ». Et voici comment Jean Bergonié, en 1923, dans son rapport devant la commission du cancer, exprime cette nécessité de distinguer le bon malade cancéreux du mauvais en ces termes de valeur sociale :

Le cancéreux que nous voyons dans ces consultations [...] est ordinairement un sujet dans toute la plénitude de son activité. Il a une valeur sociale relative quelquefois considérable : c'est un père ou une mère de famille, un ouvrier habile, un agriculteur encore solide, une femme quelque temps après la ménopause etc. Évidemment, cette valeur sociale est souvent encore plus grande chez les cancéreux des classes aisées, mais, que ce soit dans les unes ou dans les autres, on peut dire que le cancéreux au début et même pendant une assez longue période, précisément la période où il est guérissable par les moyens actuels, n'est pas un déchet social, loin de là. Et si on le conserve, ce n'est pas une non-valeur que l'on recouvre pour la société. En un mot, socialement parlant, la lutte contre le cancer peut payer.

On ne saurait le dire plus clairement : le bon malade est celui qui guérit.
Et même si les malades ne payent pas, la lutte contre le cancer peut, elle, payer : l'objectif de la cancérologie sera donc d'être rentable en guérissant. Si elle s'occupait des "incurables », elle ne serait pas rentable c'est pourquoi l'argument majeur en faveur de leur création fait référence à l'efficacité des thérapeutiques.

Contrairement à d'autres politiques publiques qui ont cours à cette époque en faveur, par exemple, de l'enfance en danger, du traitement de la syphilis ou celui de la tuberculose, il ne s'agit pas de contrôler des états pathologiques en les empêchant de s'aggraver : avec le cancer, ce sont les vertus d'une activité médicale curative qui sont mises en avant comme facteur de rentabilité sociale. Et à côté de l'« usine à guérir $"{ }^{3}$ on créera, parallèlement, des institutions charitables pour ceux que Jean-Alban Bergonié nomme les « déchets sociaux».

La commission adopte le programme de Bergonié et le Parlement vote le 30 juin 1923 une loi lui accordant ses subventions. ${ }^{4}$

\section{Figures et mésaventures du concept}

En regardant ce que la guérison fait à la cancérologie, on observe que ce qui la cadre à cette époque, c'est l'opposition curable/incurable. Or, cette distinction est d'abord une distinction de chirurgiens. Et même s'il ne faut sans doute pas trop vite traduire curable par guérissable mais plutôt par « soignable » ou traitable, Barron Lerner, historien américain, considère que c'est Halsted - chirurgien « historique » du cancer - qui a fait advenir le cancer du sein au statut de maladie guérissable en développant sa méthode de mammectomie radicale (Lerner, 2001). Cette dernière connaît un grand succès jusque dans les années 1970 avant d'être jugée totalement injustifiée - du point de vue des vies sauvées - au terme de débats et de statistiques controversées entre, notamment, féministes et chirurgiens. Dans les années 1920, la distinction curable/incurable s'appuyait de fait sur une distinction opérable/pas

3 L'expression est de Bergonié lui-même.

4 Le livre de P. Pinell (1992) et les archives de l'INH puis de l'INSERM que j'ai pu collecter montrent que la guérison est un enjeu économique et social pour la société tout entière et pas seulement pour les médecins. ("Comment doivent être organisés les centres régionaux de lutte contre le cancer”. Rapport devant la commission du cancer, Paris médical, 1923, 48.) 
opérable qui excluait une bonne partie des malades des institutions. Avant toute considération morale ou éthique sur l'exclusion des incurables du dispositif thérapeutique - celles-ci s'exprimeront après 1945 (Ménoret, 2015) - c'est le développement de la radiothérapie dans le dispositif de soins qui change la donne dans les années 1930. Les radiothérapeutes vont cesser de faire fonctionner cette catégorisation dans leur approche du malade parce qu'à la différence des chirurgiens, ils peuvent traiter à peu près tout le monde. On va ainsi progressivement sortir de cette dualité curable/incurable, comme le montre Pinell, au profit d'une certaine gradualité. Jusqu'alors, une théorie prévalait : le cancer est une maladie en deux temps. Un temps où on peut le guérir et un autre où c'est trop tard. La gradualité introduit quelques subtilités au sein de cette vision binaire au profit de pronostics plus fins. Mais comment dire, comment nommer, les nouveaux espaces d'incertitude que l'on va ainsi créer entre curable et incurable?

\section{La rémission : le concept pour l'incertitude}

Dire l'état de guérison avec certitude et sur un mode définitif s'avère très compliqué. On l'aperçoit dans la thèse de Lainé évoquée plus haut, les expressions retenues pour apprécier les résultats des thérapeutiques oscillent entre "reste intégralement guérie à cette date", "malade toujours en excellent état", "persistance de la guérison”, "une parente apporte d'elle les meilleures nouvelles", "la guérison se maintient actuellement", ou bien encore : "l'état de guérison apparent est de nouveau vérifié et se maintient trois ans et demi après le début du traitement radioactif".

On devine derrière les expressions de ces états intermédiaires des situations post-thérapeutiques où le malade n'est certes pas guéri, mais où la maladie semble cependant stoppée dans son évolution. On pourrait penser, rétrospectivement, que la rémission est le concept rêvé pour dire l'incertitude de la guérison qu'on voit pointer dans ces formulations mais, évidemment, cette catégorie n'est jamais mobilisée dans le commentaire de l'auteur à l'égard de ses statistiques puisqu'elle n'apparaît dans la littérature scientifique qu'à la fin des années 1940.
En effet, cette notion de rémission, qu'on repère pour la première fois en médecine dans un ouvrage d'Ambroise Paré en 1560, arrivera beaucoup plus tard en cancérologie. La première fois que je la vois apparaître dans mes recherches sur ce thème (Ménoret, 2002), c'est dans un article de Goodman datant de 1946. Elle apparaît avec les premiers résultats des premières chimiothérapies sur les leucémies notamment. Et elle s'installe dans le répertoire des cancérologues à partir des années 1950. La définition officielle de la rémission appliquée au champ médical c'est « disparition momentanée des symptômes ». Un entretien réalisé il y a quelques années, avec un témoin historique des premiers succès des premières chimiothérapies dans le service de Jean Bernard à l'hôpital Saint Louis à Paris, nous rappelle qu'en hématologie, pas un seul des enfants soignés ne survivait avant l'arrivée des premières chimiothérapies. Petit à petit, avec ces nouveaux traitements, on commence à recenser des cas d'amélioration qui sont porteurs d'énormément d'espoir. Parfois, l'espoir est de courte durée mais parfois il se prolonge. Qu'est-ce que la guérison dans un tel contexte?

La guérison, dorénavant on sait la calculer, la définir mais si on a fait des progrès en ce sens, en revanche on ne sait toujours pas l'attribuer avec certitude à Monsieur X ou Madame Y. C'est-à-dire qu'il est toujours incertain de passer de la définition « moyenne » à son attribution individuelle et personnelle. Parce que, aujourd'hui comme hier, on ne sait jamais comment un traitement va se terminer. Les manuels sont explicites à cet égard. La définition de la guérison est statistique : pour une population cancéreuse, on peut parler de guérison lorsque la courbe de survie de cette population devient parallèle à la courbe de survie d'une population de référence non cancéreuse, comparable en âge, sexe, condition de vie etc. et réputée normale. Pour un individu, on peut parler de guérison quand le temps écoulé sans récidive est suffisant pour rendre une récidive improbable dans le cas de la tumeur considérée. Le temps ainsi défini varie considérablement en fonction de la tumeur. L'espérance de vie ou l'espérance de guérison est l'évaluation des chances pour un patient d'être en vie à un moment déterminé ; par exemple de se trouver en vie ou guéri en apparence, dans cinq ans. 


\section{De la guérison à la survie}

On passe ainsi subrepticement de la guérison à la survie même si cette notion est difficile à mobiliser au-delà des registres, en interaction directe avec les patients. Puisque le principe binaire curable/ incurable est devenu obsolète, on va s'intéresser à la question du pronostic pour tenter de cibler des traitements en fonction des perspectives de guérison. À partir de 1942, ce sont les français qui se lancent sur ce chantier à travers l' " enquête permanente cancer » (EPC) qui pendant une dizaine d'années, jusqu'en 1952, travaille à élaborer la question pronostique avec une approche rationnelle et à sortir des intuitions empiriques. L'EPC produit en 1952 la nomenclature TNM qui permet de rationnaliser l'idée pronostique en fonction de cette classification : $\mathrm{T}$ taille de la tumeur, $\mathrm{N}$ invasion ganglionnaire et $\mathrm{M}$ métastases - et qui permet de déterminer 4 stades qui mesurent l'état de gravité de la maladie (Ménoret, 2002).

La recomposition de l'évolution du cancer en stades est une étape très importante dans le développement de la pensée médicale qui transforme le raisonnement cancérologique. La part d'incertitude inhérente à chaque cas reposait, jusqu'alors, plutôt sur des considérations empiriques plus ou moins intuitives que sur une connaissance systématique. Elle devient, dès la mise en place de la nomenclature en 1952, quantifiable et mise en probabilités.

Le commentaire épistémologique du philosophe Ian Hacking selon lequel la probabilité n'est rien d'autre que ce qui, réorganisant les savoirs, change en fait notre vision du monde s'impose ici (Hacking, 1975). En inscrivant la promesse de la guérison dans un régime de pensée probabiliste à travers un pronostic, les images attachées au cancer et aux malades amorcent un nouveau changement lié à l'usage de son nouvel outillage probabiliste.

Résumons la transformation des différents destins de prise en charge et d'images du patient à la lueur de ses promesses de guérison. D’abord, dans les années 1920, la chirurgie a fait du cancer une maladie à deux temps : l'un curable, l'autre pas. L'usine à guérir ne doit s'occuper que des malades curables, c'est sa mission publique. Les incurables sont relégués dans les institutions charitables. Assez rapidement, dans les années 1930, la population des curables s'agrandit car le critère de distinction curable/incurable ne fonctionne plus pour l'attribution de soins de radiothérapie. En 1945, le procès de Nuremberg produit une conscience éthique innovante. La volonté d'accepter tous les malades dans les centres est en effet officiellement exprimée et l'ambition d'éradication originelle va se partager avec d'autres missions : surveiller (prévention), traiter (soins), contrôler (périodes de rémission). La cancérologie se destine à des publics de plus en plus larges, la question de la guérison se pose avec de plus en plus d'acuité. L'élaboration de la Nomenclature des cancers conduit, avec la notion de stade, à penser de façon systématique le développement d'un cancer et l'avenir d'une personne malade en termes de probabilité. Si l'appréciation pronostique qui s'en dégage reste toujours inscrite dans une profonde incertitude médicale, la rémission a pour effet, sinon charge, de la nommer et de la contenir. Toute l'incertitude médicale est ramassée dans cette notion de rémission, antichambre de la guérison ou de la mort. L'incertitude quant à la guérison est la norme. Mais taboue.

En termes démographiques, les institutions anticancéreuses accueillent après la deuxième guerre mondiale de plus en plus de malades. Les discours officiels tentent d'accréditer l'idée que le cancer guérit dans plus d'un cas sur deux. En outre, là où d'aucuns souhaiteraient entendre parler de taux de guérison, c'est plutôt en termes de taux de survie que la communication se fait: le triomphe de la rhétorique de Porter (1996) trouve ici un champ d'extension. Parce que les études comptabilisent les populations qui survivent à 5 ans, ou à 10 ans, à leur cancer. Il arrive que ces chiffres soient officiellement contestés. Des polémiques ont cours sur la fiabilité des taux de guérison à partir de chiffres de survie à seulement 5 ans. Dans les années 1970/1980, le débat qui oppose les tenants de la tumorectomie seule aux tenants de la mastectomie élargie montre la fragilité de certains chiffres officiels. L'image du cancer reste sombre pour la société à cause notamment de ces taux de guérison flous pour le grand public dont l'incertitude est réalimentée par des débats sibyllins qui restent d'ailleurs la plupart du temps réservés aux professionnels. Dans son combat en faveur de la guérison des cancers, l'État met en œuvre des politiques de santé publique censées résoudre une 
mortalité persistante. La prévention s'inscrit dans ces carences du curatif. Prévention et dépistage se développent, dans les discours et dans les faits. Les autorités considèrent que les politiques de dépistage pourraient être mieux suivies si l'image du cancer était améliorée c'est-à-dire si on - le grand public, les profanes - croyait un peu plus à la guérison. Régulièrement des campagnes sont organisées sur ce thème depuis l'entre-deux-guerres. Une d'entre elles retient l'attention sur notre sujet. En 2007, l'INCa se voit attribuer la tâche de s'attaquer aux représentations négatives liées au cancer. Et c'est ainsi que naît la notion de « héros ordinaire ».

\section{Oxymore de guérison}

Car on a toujours beaucoup de mal à parler des personnes guéries, 84 ans après l'« usine à guérir ". Comment les appeler? Comment les mettre en scène et en valeur? En 2007, l'institut national du cancer français, l'INCa, invente les « héros ordinaires » pour les désigner. L'argument exprimé dans le dossier de presse qui présentait cette campagne en faveur d'une meilleure image du cancer précise pourquoi l'expression «héros ordinaires » a été préférée à celle de « survivant» et retenue. Dans les termes suivants : «Aux États-Unis, on les appelle les « survivors » et ils revendiquent haut et fort leur appartenance à cette communauté qu'ils n'ont pas choisie. Comme John Wayne ou Lance Amstrong, ils contribuent à lever le silence qui pèse encore sur cette maladie. En France, il fallait trouver un terme approprié [c'est moi qui souligne]. C'est ainsi à dessein qu'ont été assemblés deux mots désignant des réalités contradictoires, « héros » et « ordinaire ».»

Donc, en français, ou en France plus exactement, le terme approprié à « survivor », pour les communicants du cancer mais aussi pour les institutions qui les font travailler bien entendu, c'était donc en 2007 « héros ordinaires".

Entre l'usine à guérir et le héros ordinaire : 84 années de difficulté à dire qui est guéri du cancer, à dire la guérison des personnes ayant eu un cancer. Derrière toutes ces difficultés, une question : est-ce qu'on est jamais guéri d'un cancer? En filigrane c'est cette question qui est contenue dans cet indicible. Et cet indicible est aussi un invisible dans la mesure où cette opacité se perpétue sur les populations concernées aujourd'hui encore.

\section{Par-delà maladie et guérison}

Pour qu'un groupe social existe, il faut qu'il soit identifié. Les sciences sociales l'ont très peu fait jusqu'à présent en France et on ne sait même pas nommer le temps de l'après-cancer. On connaît les caractères performatifs du langage. S'il n'existe aucun mot pour dire le statut de cet après en France, la situation est fort différente de l'autre côté de l'Atlantique et de la Manche.

\section{Une seule assurance : l'incertitude, un seul état : survivant.e}

En Juillet 1985, paraît dans le New England Journal of Medicine un papier intitulé "Seasons of Survival: Reflections of a Physician with Cancer". Ce papier très personnel signé du Dr Fitzhugh Mullan esquisse publiquement le début d'un changement médical et culturel important aux Etats-Unis. Il développe en effet l'idée que tout patient atteint d'un cancer est bien plus qu'un patient : il est justement un survivant, ce titre sur lequel le français renâcle. Pour Mullan, les statuts de maladie et guérison sont inadéquats pour le cancer car ils ne parviennent pas à rendre compte de l'incertitude qui va marquer de manière irréversible chaque existence de personne qui se verra diagnostiquée pour une affection cancéreuse. C'est la raison pour laquelle il adopte cette notion de «survie», afin de définir la condition d'incertitude commune par laquelle passent toutes les personnes qui ont à lutter un jour (lutter toujours ?) contre cette maladie. L'état de survie et, partant, le statut de survivant, selon le Dr Mullan, commencent au moment du diagnostic, lorsque les personnes sont obligées de se confronter à leur propre mortalité et de commencer à s'ajuster à ce qui va constituer, non seulement leur existence immédiate, mais également leur avenir à plus long terme.

Son article propose un changement majeur d'affectation de la notion de survie dans le registre des mots du cancer. Car cette notion résidait jusqu'alors dans les travaux quantitatifs sans troubler particulièrement les esprits. Les chercheurs 
produisaient et distribuaient depuis de longues années de nombreuses statistiques en termes de «taux de survie », articulant de fait degré de sévérité de la maladie et pronostic. Mullan, en insistant sur la caractéristique d'incertitude majeure qui distingue tout diagnostic de cancer, est le premier à rendre compte de l'expérience de cette maladie en termes de survie et la personne qui a ou qui a eu un cancer en tant que survivant.e. La notion de taux de survie était somme toute banale, confinée dans ce registre épidémiologique, mais le passage de cette notion vers un statut à la fois beaucoup plus qualitatif et personnalisable va engendrer des retombées insoupçonnables.

\section{Institutionnalisation de l'incertitude}

En 1986, Mullan participe à la fondation de la National Coalition for Cancer Survivorship. Progressivement, dans les années 1990, cette nouvelle représentation du cancer, notamment temporelle, permet d'établir de nouveaux cadres de réflexion et d'action : tant pour la recherche en santé que pour les politiques initiées alors. En 1995 , par exemple, le National Cancer Institute parraine le premier National Congress on Cancer Survivorship et, en 1996, crée l'Office of Cancer Survivorship. Des organisations telles que l'American Cancer Society ou l'American Institute for Cancer Research donnent priorité à la notion de survie dans leur programme de recherche. Pendant cette période, le taux de survie représente un domaine renforcé de préoccupation pour la recherche et le survivant du cancer est constitué comme un objet d'étude scientifique (King, 2006). L'augmentation du nombre de survivants à un cancer et leur visibilité accrue vont désormais déterminer une redéfinition importante des soins, avec des professionnels de santé qui, notamment mettent l'accent de manière inédite sur les effets tardifs à long terme de diagnostic et de traitement.

Les Centers for Disease Control and Prevention, mais également la puissante Fondation Lance Armstrong, prennent une avance symbolique dans ces efforts, en mettant en œuvre un National Action Plan for Cancer Survivorship en 2004. En 2006, l'Institute of
Medicine of the National Academies publie un rapport conséquent sur le sujet, présentant des protocoles susceptibles d'améliorer les soins de longue durée et la qualité de vie des survivants. Aujourd'hui, le vocabulaire de survie est partout : sites web, groupes de soutien, associations, publications scientifiques, officielles, personnelles etc. Aux Etats-Unis, être un patient atteint de cancer revient à être un survivant du cancer. En d'autres termes, avoir un cancer à un moment donné se traduit ontologiquement avec le fait d'être identifié à son caractère d'infinitude.

Accepter de se définir ou d'être défini sous ce vocable suppose donc plus ou moins implicitement l'approbation d'une similitude de destin au-delà d'expériences forcément différenciées selon les individus et/ou les types de cancer. Cela implique également que l'on accepte d'affirmer un lien essentiel et permanent entre soi et sa maladie. Ceci transforme tous les types de cancers en une seule maladie lisible. Et, last but not least, marque une identité irrémédiablement, perpétuellement et infiniment en termes de survivant. Le concept de survie ainsi élaboré rencontre bien entendu des critiques à la fois médicales et populaires (Baszanger, 2009).

\section{Les enjeux du " connaître l'après »}

Faire exister socialement, médicalement, scientifiquement les survivants, c'est se donner les moyens de mieux les connaître pour mieux les traiter. Notamment. C'est par exemple rendre visibles des effets préjudiciables à long terme. Au niveau social : les situations de travail des ex-malades sont assujetties à beaucoup de discriminations mal identifiées (VICAN 1 et $2^{5}$ ) (Inca, 2014; Le CorollerSoriano; Malavolti; Mermilliod, 2008).

$\mathrm{Au}$ niveau médical et scientifique : plus les thérapies sont intensives et plus les effets délétères sont susceptibles d'être forts dans la longue durée. Ces effets sont plus ou moins graves en termes de pronostic vital mais ils sont toujours invalidants. Pour l'instant, ils sont rarement pris au sérieux par les professionnels (Abel; Subramanian, 2008). Les effets secondaires immédiats sont traités mais à plus

\footnotetext{
5 L'étude VICAN porte sur les conditions de vie des personnes atteintes, un ou deux ans après un diagnostic de cancer. VICAN 2 a été réalisée en 2014 par l'Institut national du cancer, en partenariat avec l'Inserm et les trois principaux régimes d'assurance maladie.
} 
long terme, dans la durée, les choses se compliquent. Et à moyen ou long termes, certains de ces effets vont être d'autant plus difficiles à vivre qu'on ne va pas leur accorder l'intérêt qu'ils méritent. Ce constat est valable pour la plupart des cancers. Il est surtout documenté pour le cancer du sein qui éclaire quelques conditions d'existence des ex-patient.e.s.

Des thèmes connus et reconnus reviennent dans la liste des symptômes dont font part les femmes après leur cancer du sein : douleur, problèmes de lymphœdème, ménopause prématurée, problèmes de sexualité, de libido, problèmes de reconstruction, de prothèse etc... Ces problèmes sont identifiés et pris en charge relativement systématiquement dans la phase aiguë ou juste après. À côté de ceux-là, il y en a d'autres, qui persistent, qui sont plus sournois et qui reçoivent beaucoup moins d'attention. Les symptômes que les femmes rapportent le plus souvent de leur condition chronique sont par exemple la fatigue ou des déficiences cognitives (problèmes de mémoire, de concentration etc.). Et comme ces symptômes peuvent difficilement être vérifiés, ils sont en général assez peu validés.

De fait, «survivants » et " héros ordinaires » auraient tout intérêt à être identifiés et analysés en étant réinsérés dans leurs conditions collectives et politiques plutôt qu'isolés dans une dimension strictement individuelle. À être pensés aussi dans leurs dimensions économique, politique et sociale (Ménoret, 2006). Parce qu'ils se retrouvent régulièrement, 10 ans, 20 ans après leur cancer, dans le cabinet d'un médecin qui ne connaît rien de leurs traitements passés : lequel, les entretiens de mon enquête « survivre à un cancer » le confirment, rate la plupart du temps (et ce pour une durée plus ou moins longue) l'étiologie de la séquelle iatrogène, avec un dossier médical qui n'a pas été saisi dans la durée de la trajectoire de maladie de la personne. C'est ce qu'ont compris les cancérologues contemporains d'oncologie pédiatrique qui s'intéressent aujourd'hui au devenir de leurs jeunes patients. Pourquoi sont-ils les seuls ? Car l'intérêt des professionnels pour les effets possiblement iatrogènes de leurs traitements anticancéreux se manifeste tard et de manière extrêmement ciblée.

Plusieurs hypothèses peuvent être retenues pour expliquer ce phénomène de méconnaissance entretenue. D'abord parce que la faible accumulation et donc la faible diffusion des connaissances sur ces effets iatrogènes à long terme repose entre autres sur le fait que les essais cliniques randomisés et les grandes cohortes demeurent les formes de preuve dominantes pour les autorités publiques et la profession médicale. Or, ces essais sont non seulement longs et coûteux à établir mais, de surcroît, financés par les entreprises pharmaceutiques. Pour ces compagnies il s'agit évidemment, afin de gagner le marché, de prouver que leurs médicaments sont non seulement efficaces mais aussi qu'ils sont safe. Pour obtenir leur autorisation de mise sur le marché, les essais cliniques en toute logique commerciale mesurent plus les taux de survie à 5 ans que les effets secondaires à long terme.

Ensuite, concernant la faible diffusion des savoirs produits sur le sujet, on remarque que d'autres savoirs sont produits ailleurs - c'est le cas de toute une littérature de SHS consacrée aux survivors avec, notamment, l'existence d'une revue scientifique qui leur est consacrée, Journal of Cancer Survivorship mais leur diffusion est limitée, notamment en France.

Enfin, la question du maintien tacite d'une forme de secret, auprès des populations concernées, à propos des risques iatrogènes qu'ils encourent après le traitement qui les a tirés d'affaire mérite d'être posée. Dans de telles circonstances, plusieurs réponses à cette question ont été apportées, différentes et complémentaires. Proctor (2012), dans sa recherche à propos de Big Tobbaco, considère que le public est laissé dans l'ignorance de son empoisonnement afin de préserver des intérêts institutionnels ou marchands. Fillion et Torny (2016) dans leur approche du DES (Distilbène ${ }^{\circledR}$ ), émettent l'hypothèse que l'ignorance programmée des profanes est vue par ceux qui en décident comme une forme de protection. Dans ce cas, la connaissance des profanes est envisagée plutôt comme une source de panique que comme une source d'action raisonnée. Cette vision en dit long sur le degré d'immaturité intellectuelle attribué aux non-professionnels.

Dans le cas de la cancérologie, on peut sans doute ajouter des sources intentionnelles d'ignorance liées au déni des professionnels. Notamment pour des médecins qui se trouveraient dans cette posture face aux dégâts de leurs anciennes thérapies et dans l'impossibilité de faire face à des erreurs passées. 
Quoi qu'il en soit, héros ordinaires ou survivants du cancer pourraient avoir tout intérêt à être analysés en étant réinsérés dans leurs conditions collectives d'existence. Pour exister socialement d'une part et pour, d'autre part, voir les problèmes sanitaires et sociaux qui les caractérisent reconnus. On dit souvent qu'on ne sait jamais quand débute un cancer mais sait-on jamais quand il se termine? La méconnaissance à propos de la guérison est parfois délétère. Et en tout cas jamais neutre.

\section{Références}

ABEL, E. K.; SUBRAMANIAN, S. K. After the cure. New York: New York University Press, 2008.

BASZANGER, I. Between "survivorship" and "living with dying": recreating normality in oncology. In: DRUGS, STANDARDS, AND CHRONIC ILLNESS, 2009, Manchester. Programme... Manchester: BSHS, 2009.

BERLIVET, L. Les ressorts de la biopolitique: dispositifs de sécurité et processus de subjectivation au prisme de l'histoire de la santé. Revue d'Histoire Moderne et Contemporaine, Paris, v. 13/4, n. 6o-4/4 bis, p. 97-121, 2013.

CANGUILHEM, G. Une pédagogie de la guérison est-elle possible? In: CANGUILHEM, G. Écrits sur la Médecine. Paris: Seuil, 2002. p. 69-99.

CARRICABURU, D.; MÉNORET, M. Sociologie de la santé: institutions, professionnels et maladies. Paris: Armand Colin, 2004.

FILLION, E.; TORNY, D. Un précédent manqué: le Distilbène ${ }^{\circledast}$ et les perturbateurs endocriniens: contribution à une sociologie de l'ignorance. Sciences Sociales et Santé, London, v. 34, n. 3, p. 4775, 2016.

HACKING, I. The emergence of probability. Cambridge: Cambridge University Press, 1975. HERGÉ. L'affaire tournesol. Tournai: Casterman, 1956.

INCA - INSTITUT NATIONAL DU CANCER. La vie deux ans après un diagnostic de cancer: de l'annonce à l'après cancer. Boulogne Billancourt, 2014.
KING, S. Pink Ribbons, Inc.: breast cancer and the politics of philanthropy. Minneapolis: University of Minnesota Press, 2006.

LAINÉ, R. Le Centre Régional de Lutte contre le Cancer de Nantes: 1924-1929. 1929. Tese (Doutorado em Medicina) - Faculté de Médecine de Paris, Paris, 1929.

LE COROLLER-SORIANO, A. G.; MALAVOLTI, L.; MERMILLIOD, C. (Dir.). La vie deux ans après le cancer. Paris: La Documentation Française, 2008.

LERNER, B. The breast cancer wars. Oxford: Oxford University Press, 2001.

MÉNORET M. Les temps du cancer. Paris: Editions du CNRS, 1999.

MÉNORET, M. Genesis of the notion of stage in oncology: the French 'Enquête Permanente Cancer' (1943-1952). Social History of Medicine, Oxford, v. 15 , n. 2, p. 291-302, 2002.

MÉNORET, M. Prévention du cancer du sein: cachez ce politique que je ne saurais voir. Nouvelles Questions Féministes, Berna, v. 26, n. 2, p. 32-47, 2006.

MÉNORET, M. La prescription d'autonomie en médecine. Anthropologie et Santé, Paris, n. 10, 2015.

MULLAN, F. Seasons of survival: reflections of a physician with cancer. New England Journal of Medicine, Boston, v. 313, p. 270-273, 1985.

PINELL, P. Naissance d'un fléau: histoire de la lutte contre le cancer en France (1890-1940). Paris: Métailié, 1992.

PORTER, T. Trust in numbers: the pursuit of objectivity in science and public life. Princeton: Princeton University Press, 1996.

PROCTOR, R. N. Golden holocaust: origins of the cigarette catastrophe and the case for abolition. Berkeley: University of California Press, 2012.

RETTIG, R. A. Cancer crusade: the story of the National Cancer Act of 1971. Princeton: Princeton University Press, 1977.

Reçu: 21/05/2018

Approuvé: 08/08/2018 